Please do not remove this page

RMIT

UNIVERSITY

\title{
Investigation of the mechanisms of electromagnetic field interaction with proteins
}

Vojisavljevic, Vuk; Pirogova, Elena; Cosic, Irena

https://researchrepository.rmit.edu.au/esploro/outputs/9921858790201341/filesAndLinks?institution=61RMIT_INST\&index=null

Vojisavljevic, V., Pirogova, E., \& Cosic, I. (2005). Investigation of the mechanisms of electromagnetic field interaction with proteins. Proceedings of the 27th Annual International Conference of the IEEE

Engineering in Medicine and Biology Society. https://doi.org/10.1109/IEMBS.2005.1616257

Published Version: https://doi.org/10.1109/IEMBS.2005.1616257

Repository homepage: https://researchrepository.rmit.edu.au

(c) 2005 IEEE. Personal use of this material is permitted. However, permission to reprint/republish this material for advertising or promotional purposes or for creating new collective works for resale or redistribution to servers or lists, or to reuse any copyrighted component of this work in other works must be obtained from the IEEE.

Downloaded On 2023/04/27 01:00:26 +1000 


\title{
Investigation of the Mechanisms of Electromagnetic Field Interaction with Proteins
}

\author{
Vuk Vojisavljevic, Elena Pirogova, Irena Cosic , Member, IEEE
}

\begin{abstract}
In our earlier work we have proposed that protein activation is electromagnetic in its nature. This prediction is based on the Resonant Recognition Model (RRM) where proteins are analyzed using digital signal processing (DSP) methods applied to the distribution of free electron energies along the protein sequence. This postulate is investigated here by applying the electromagnetic radiation to example of $\mathrm{L}$ Lactate Dehydrogenase protein and its biological activity is measured before and after the exposures. The concepts presented would lead to the new insights into proteins susceptibility to perturbation by exposure to electromagnetic fields and possibility to program, predict, design and modify proteins and their bioactivity.
\end{abstract}

\section{INTRODUCTION}

E xperimental investigations of the interaction of EMF and biosystems remain intensely controversial - where some research concerns with ELF effects and others with RF exposures. The ideas of resonant absorption and resonant interactions have been proposed as an explanation for the marked sensitivity of living systems to EMFs.

Each biological process involves a number of interactions between proteins and their targets. These interactions are based on the energy transfer between the interacting molecules. Protein interactions are highly selective, and this selectivity is defined within the protein structure. However, the physical nature of these interactions is not yet well understood. The most acceptable among the existing models is the so-called key-and-lock model, which is based on a spatial complementarity between the interacting molecules. However, with knowledge of more 3-D structures of proteins and their complexes with ligands it can be observed that the spatial complementarity is not selective enough to be considered as a sole parameter able to describe the nature of protein interactions occurred in living systems.

There is much evidence that biological processes can be induced or modulated by induction of light of particular

Manuscript submitted 8 April, 2005. This work was supported by the National Australian Centre of Radiofrequency Bioeffects Research (ACRBR) and RMIT University's Research Investment Fund.

V. Vojisavljevic is with School of Electrical and Computer Engineering, RMIT University, GPO Box 2476V, Melbourne 3001 VIC Australia and with Australian Centre of Radiofrequency Bioeffects Research (ACRBR), Australia (phone: +61 3 9925-3077; fax: +61 3 9925-2954; e-mail: naturoved@netscape.net).

E. Pirogova is with School of Electrical and Computer Engineering, RMIT University, GPO Box 2476V, Melbourne 3001 VIC Australia and with Australian Centre of Radiofrequency Bioeffects Research (ACRBR), Australia (e-mail: elena.pirogova@rmit.edu.au).

I. Cosic is with School of Electrical and Computer Engineering, RMIT University, GPO Box 2476V, Melbourne 3001 VIC Australia and with Australian Centre of Radiofrequency Bioeffects Research (ACRBR), Australia (e-mail: irena.cosic@rmit.edu.au). characteristic frequencies [1]-[6]. This is caused directly by the light-induced changes of energy states of proteins. The function of some proteins is directly connected with the absorption of visible light of defined wavelengths (e.g. rhodopsins). In addition, there is evidence that light of a defined frequency can induce or enhance some biological processes, which are normally controlled by proteins only (i.e. cell growth and proliferation [2]-[6]). All these frequency selective effects of light on biological processes of protein activation imply that protein activation involves energies of the same order and nature as the electromagnetic irradiation of light.

It has been shown in our previous research that all protein sequences with the common biological function have common frequency component in the distribution of free energy of electrons along the protein backbone. [7], [8]. This characteristic frequency is related to the protein biological function as it was found in our previous investigations [7][11]. Furthermore, it was shown that the proteins and their targets have the same characteristic frequency in common. Thus, it can be postulated that the RRM frequencies characterize not only a general function but also a recognition and interaction between the particular protein and its target at the distance. Thus, protein interactions can be considered as resonant energy transfer between the interacting molecules. This energy can be transferred through oscillations of a physical field, possibly electromagnetic in nature [7], [8].

This paper presents the results of our preliminary study of investigation the possibility of EMF radiation to modify a bioactivity of the selected enzyme.

\section{METHODOLOGY}

Enzymes are proteins crucial in accelerating metabolic reactions in the living organism. Dehydrogenases are the enzymes that catalyze a variety of oxidation-reduction reactions within the cells. In this study we have performed the structure-function analysis of dehydrogenases using the Resonant Recognition Model (RRM), which is based on DSP methods, Fourier and Wavelet Transform, applied to the proteins sequence [7], [8]. The application of the RRM in structure/function analysis of protein molecules involves several stages of calculation.

Firstly, by assigning the Electron-Ion Interaction Potential (EIIP) [7] to each amino acid, the protein sequence is represented as a series of discrete data. Then the numerical series obtained are analyzed by digital signal analysis methods, Fourier and Wavelet transform, in order to extract information pertinent to the biological function. A multiple 
cross-spectral function is defined and calculated to obtain the common frequency components from the spectra of a group of proteins. Peaks in such function denote common frequency components for all sequences analyzed.

Proteins and DNA have certain conducting or semiconducting properties [1]-[6], [12]. Consequently, a charge moving through the molecule's backbone and passing different energy stages caused by different side groups of various amino acids or nucleotides, provides sufficient conditions for the emission of electromagnetic waves. Their frequency range depends on charge velocity, which then depends on the nature of charge movement (superconductive, conductive, soliton transfer, etc) and on the energy of the field that causes this charge transfer. The nature of this physical process is still unknown. There are some models accepted that reveal the possibility, however, the complexity of the system does not allow precise calculations of charge velocity.

In the RRM [7], [8], despite the fact that conductive electron transfer at the $\mathrm{N}$ - and C-terminals of the protein is beset by not well understood conditions, the potential energy difference is:

$$
\mathrm{W}=\mathrm{W}(\mathrm{COOH})-\mathrm{W}(\mathrm{N} \mathrm{H} 2)=0.13 \mathrm{Ry}
$$

This energy difference allows for a maximum velocity of the electrons is

$$
\mathrm{V}_{\text {max }}=\sqrt{ }(2 \mathrm{eW} / \mathrm{m})
$$

where $\mathrm{e}$ is the electron charge, and $\mathrm{m}$ is electron mass. Therefore

$$
\mathrm{V}<7.87 \times 10^{5} \mathrm{~m} / \mathrm{sec} \text {. }
$$

An inherent assumption is that amino acids in the protein are equidistant and the distance is $\mathrm{d}=3.8 \AA$. Therefore, the maximum frequency that could be emitted during the electron transfer is

$$
\text { Fmax }<\mathrm{V} /(2 \mathrm{~d})<1 \times 10^{15} \mathrm{~Hz}
$$

while the corresponding wavelength is $\operatorname{Lmin}>330 \mathrm{~nm}$. The minimum frequency that could be emitted depends on the total length of the protein,

$$
\mathrm{Fmin}=2 \mathrm{Fmax} / \mathrm{N}<1 \times 10^{13} \mathrm{~Hz}
$$

where $\mathrm{N}$ is the total number of amino acids in the protein. For example, for proteins of 200 a a. in length, the minimum frequency is Fmin and the corresponding wavelength is $\mathrm{Lmax}<30000 \mathrm{~nm}$ [7]. The range from 30000 $\mathrm{nm}$ to $300 \mathrm{~nm}$ is very wide, starting from the far infrared through the visible to the ultraviolet regions.

The characteristic frequency identified for a particular protein group characterizes one particular biological function/interaction. It was postulated that the protein function is directly related to the absorption of light of defined wavelength. Within the RRM it was found that a strong linear correlation exists between the predicted and experimentally determined frequencies corresponding to the absorption of electromagnetic radiation of such proteins [7], [8]. It is inferred that approximate wavelengths in real frequency space can be calculated from the RRM characteristic frequencies for each biologically related group of sequences. These calculations can be used to predict the wavelength of the light irradiation, which might affect the biological activity of proteins exposed [7], [8], [12].

Our experimental study consists of the series of experiments that can confirm possibility that protein activity can be influenced by external electromagnetic radiation as predicted by the RRM. Here we have monitored the chemical process involving L-Lactate Dehydrogenase (rabbit muscle) by analysis of the change in its kinetics under the influence of external EMF radiation. L-Lactate dehydrogenase (LDH) catalyses the following reaction: $\mathrm{LDH}$

Pyruvate + NADH ------> Lactate $+\mathrm{NAD}^{+}+\mathrm{H}^{+}$(7)

There are several different forms of $\mathrm{LDH}$ found in the body with different kinetic properties. These forms of the enzyme are referred to as isozymes. The "M" form, of $\mathrm{LDH}$ was found in anaerobic tissue, while the " $\mathrm{H}$ " form is found in highly aerobic tissues, such as the heart. $\mathrm{H}$ form has an effective lower turnover rate for pyruvate, which assists to shunt pyruvate into the mitochondria and hence into the Krebs cycle. The suitability of LDH enzyme for this reaction is attributed to the absorption characteristics of $\mathrm{NADH}$ (Nicotinamide Adenine Dinucleotide, Reduced form). $\mathrm{NADH}$ is able to absorb the light at $340 \mathrm{~nm}$ in contradict to the NAD (Nicotinamide Adenine Dinucleotide Nicotinamide Adenine Dinucleotide, Oxidized form), which is inactive at this frequency. Due to the different optical characteristics of NADH and NAD we are able to optically assess if the reaction Pyruvate $\rightarrow$ Lactate in the presence of $\mathrm{LDH}$ as an accelerator has occurred and then determine amount of the reactants. The reaction rate depends on the concentration of enzyme and substrate.

\section{Equipment}

Monochromator Spex 270 (Instruments CA, Inc), range 400$890 \mathrm{~nm}, \mathrm{RS} 232$ connection with HP 34001A, controlled by LabView 6.1 (National Instruments). Also used will be Spectrometer USB2000 coupled with USB-ISS-UV/VIS (Ocean Optics, Inc.), range 190nm-870nm, CCD detector with 2048 pixels, USB-2 connection with Pentium IV (Windows XP), controlled with OOIBase32 software. Software automatically monitors and saves the absorption coefficient at $340 \mathrm{~nm}$ every $30 \mathrm{sec}$.

\section{MATH}

In our previous work [13] we analyzed 72-dehydrogenase protein sequences using the RRM approach. A multiple cross-spectral analysis was performed resulting in one prominent frequency identified at $\mathrm{f}=0.1680$ with $\mathrm{S} / \mathrm{N}=511.1$ (Fig.1). The presence of only one prominent peak with the significant $\mathrm{S} / \mathrm{N}$ ratio in a consensus spectrum implies that all of the analyzed sequences within the group have this frequency component in common. This frequency is related to the biological activity as it was found in our previous investigations [7], [8], [13].

As mentioned above a linear correlation between the absorption spectra of proteins and their RRM spectra with a 
regression coefficient of $\mathrm{K}=201$ and predetermined frequency range was established [7], [8]. Thus, based on the characteristic frequency determined for the whole dehydrogenase functional group, we can calculate the wavelength of irradiation, $\lambda$, which assumingly would activate protein sequences and modify their bioactivity:

$$
\lambda=201 / f_{\text {RRM }} \text {. }
$$

The dehydrogenases' characteristic frequency is at $\mathrm{f}=0.1680$; the wavelength of the electromagnetic exposure required for dehydrogenase enzymes activation is $1196 \mathrm{~nm}$.

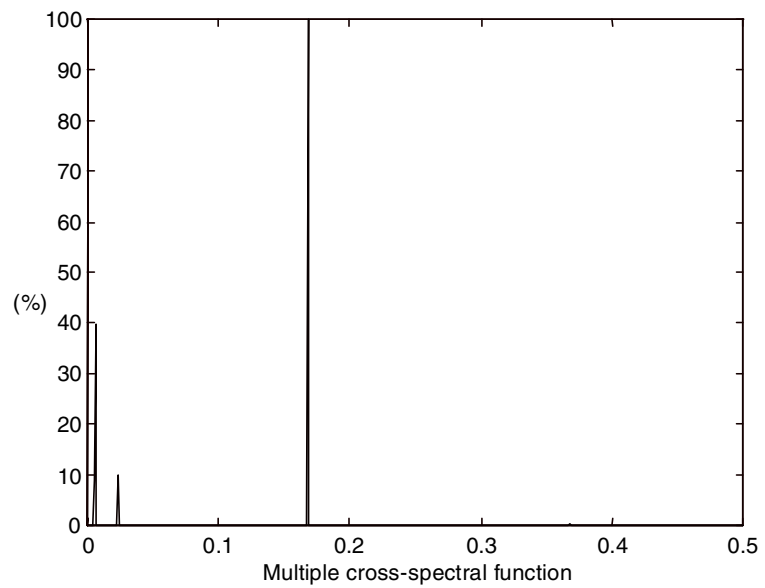

Fig. 1 Multiple cross-spectral function of Dehydrogenase proteins (72 sequences). The prominent peak(s) denote common frequency components. The abscissa represents RRM frequencies, and the ordinate is the normalized intensity.

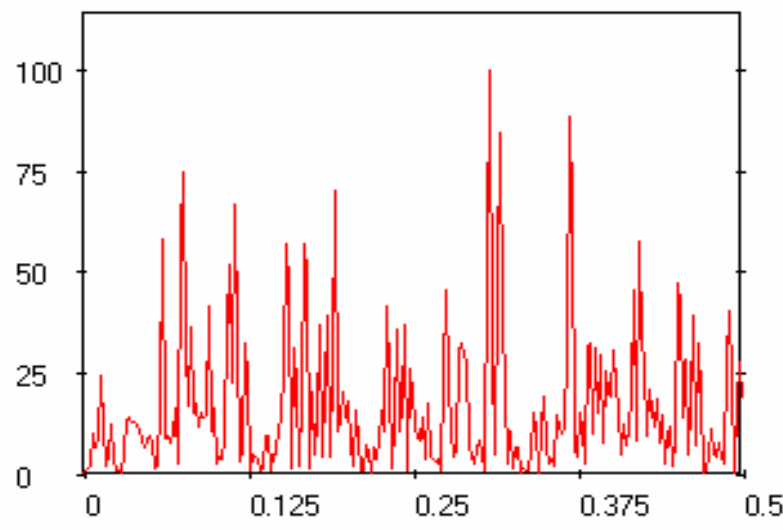

Fig. 2 Single spectrum of LDH (1.1.1.27 rabbit muscle)

In this study, however, we cannot irradiate the selected enzyme sample by EMF of this required wavelength due to the limitations of Monochromator Spex 270 (Instruments CA, Inc) with a range of $400-890 \mathrm{~nm}$. To solve this problem we decided to look at the single spectrum of the studied LDH enzyme (1.1.1.27 rabbit muscle) that is shown in Fig.2. The RRM characteristic frequency of this enzyme is identified at $\mathrm{f}_{\mathrm{RRM}}=0.3066$ that corresponds to $\lambda=656 \mathrm{~nm}$. Therefore, to test the concept of the possible affect of EMF on enzyme activity here we are used the external radiation in a range of 550-850.
Measurement of the absorption spectrum of NADH:

1. We diluted the stock coenzyme solution with the $0.003 \mathrm{M}$ potassium phosphate assay buffer. 2. Using the properly diluted coenzyme solution, we measured the absorbance at $25-50 \mathrm{~nm}$ intervals between 550 and $850 \mathrm{~nm}$. 3. The spectrophotometer is se $\mathrm{t}$ to $100 \%$ transmittance (zero absorbance) at each wavelength using the $0.003 \mathrm{M} \mathrm{K}_{2} \mathrm{HPO}_{4}$ assay buffer blank.

The results obtained have shown that NADH concentration corresponds to the maximum absorbance of 1.2 at $340 \mathrm{~nm}$. Fig. 3 shows how NADH sample absorbance is affected by the applied radiation of the defined wavelength.

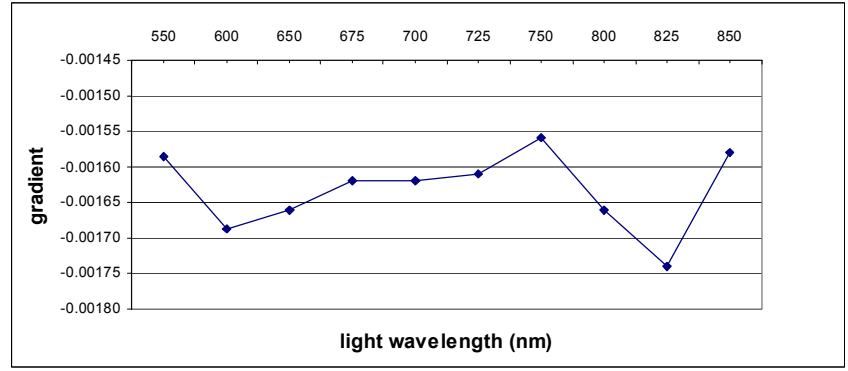

Fig. 3 Gradient of change in absorbance of NADH

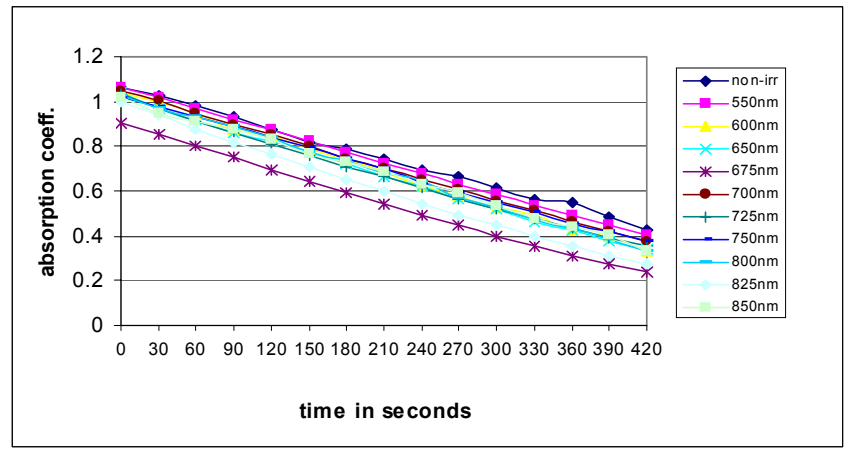

Fig. 4 Changes of absorption coefficient values (at $340 \mathrm{~nm}$ ) in time after irradiation with different wavelengths

Measurement of LDH activity

$2.5 \mathrm{ml}$ cuvettes are filled with the following components:

1. $0.1 \mathrm{ml}$ of $0.0027 \mathrm{M}$ Sodium Pyruvate (BioWhittaker)

2. $0.1 \mathrm{ml} \mathrm{NADH}$, disodium salt, $(\mathrm{C} 21 \mathrm{H} 27 \mathrm{~N} 7 \mathrm{O} 14 \mathrm{P} 2 \mathrm{Na} 2)$

(Roche), 0.005M Phosphate buffered saline (SIGMA)

3. $1.7 \mathrm{ml}$ deionized water

4. $0.3 \mathrm{ml} \mathrm{LDH}$ diluted in $2.5 \mathrm{~g} / \mathrm{ml}$ of Phosphate buffered saline with BSA (SIGMA).

Experiments were performed at room temperature $24^{\circ} \mathrm{C}$. The cuvettes were filled with $0.3 \mathrm{ml}$ of the LDH samples. The samples were previously irradiated with the light of different wavelengths for $10 \mathrm{~min}$. These irradiated samples were added to the already prepared solution of NADH and pyruvate. The samples were previously irradiated with the light different 
ABSORBANCE AND WAVELENGTH VALUES OF NADH SAMPLE

\begin{tabular}{|c|c|c|c|c|c|c|c|c|c|c|c|}
\hline sec & non-irr & $550 \mathrm{~nm}$ & $600 \mathrm{~nm}$ & $650 \mathrm{~nm}$ & $675 \mathrm{~nm}$ & $700 \mathrm{~nm}$ & $725 \mathrm{~nm}$ & $750 \mathrm{~nm}$ & $800 \mathrm{~nm}$ & $825 \mathrm{~nm}$ & $850 \mathrm{~nm}$ \\
\hline 0 & 1.061 & 1.064 & 1.042 & 1.04 & 0.906 & 1.05 & 1.032 & 1.016 & 1.026 & 0.997 & 1.016 \\
\hline 30 & 1.027 & 1.02 & 0.997 & 0.966 & 0.855 & 1.004 & 0.966 & 0.977 & 0.968 & 0.937 & 0.95 \\
\hline 60 & 0.98 & 0.971 & 0.946 & 0.919 & 0.804 & 0.95 & 0.914 & 0.93 & 0.933 & 0.878 & 0.91 \\
\hline 90 & 0.93 & 0.921 & 0.873 & 0.859 & 0.75 & 0.9 & 0.862 & 0.886 & 0.891 & 0.818 & 0.877 \\
\hline 120 & 0.876 & 0.872 & 0.846 & 0.827 & 0.697 & 0.851 & 0.811 & 0.838 & 0.838 & 0.764 & 0.828 \\
\hline 150 & 0.818 & 0.824 & 0.786 & 0.77 & 0.646 & 0.802 & 0.761 & 0.796 & 0.777 & 0.706 & 0.769 \\
\hline 180 & 0.785 & 0.772 & 0.735 & 0.722 & 0.593 & 0.747 & 0.711 & 0.743 & 0.739 & 0.651 & 0.728 \\
\hline 210 & 0.744 & 0.721 & 0.689 & 0.664 & 0.543 & 0.7 & 0.663 & 0.701 & 0.682 & 0.597 & 0.689 \\
\hline 240 & 0.692 & 0.678 & 0.621 & 0.62 & 0.493 & 0.653 & 0.615 & 0.633 & 0.642 & 0.544 & 0.631 \\
\hline 270 & 0.664 & 0.63 & 0.575 & 0.562 & 0.445 & 0.606 & 0.567 & 0.59 & 0.57 & 0.492 & 0.592 \\
\hline 300 & 0.618 & 0.582 & 0.531 & 0.519 & 0.398 & 0.558 & 0.523 & 0.547 & 0.527 & 0.445 & 0.535 \\
\hline 330 & 0.567 & 0.536 & 0.488 & 0.464 & 0.353 & 0.51 & 0.477 & 0.506 & 0.473 & 0.396 & 0.479 \\
\hline 360 & 0.546 & 0.493 & 0.43 & 0.424 & 0.312 & 0.464 & 0.434 & 0.457 & 0.435 & 0.351 & 0.442 \\
\hline 390 & 0.483 & 0.449 & 0.394 & 0.375 & 0.274 & 0.418 & 0.392 & 0.419 & 0.386 & 0.31 & 0.403 \\
\hline 420 & 0.429 & 0.406 & 0.335 & 0.337 & 0.241 & 0.376 & 0.353 & 0.373 & 0.335 & 0.272 & 0.335 \\
\hline Gradient & -0.00149 & -0.00159 & -0.00169 & -0.00166 & -0.00162 & -0.00162 & -0.00161 & -0.00156 & -0.00166 & -0.00174 & -0.00158 \\
\hline
\end{tabular}

wavelengths for $10 \mathrm{~min}$. These irradiated samples were added to the already prepared solution of NADH and pyruvate. The absorption coefficients were measured at $340 \mathrm{~nm}$. We repeated the above measurements twice for each wavelength of the applied irradiation. The results obtained are shown in Table 1 and Fig. 4.

\section{DISCUSSION}

The results of this preliminary study clearly demonstrated the change of absorbance of NADH sample under the influence of irradiated LDH. After being exposed to EMF in the range of 550-850nm, the LDH bioactivity has increased resulting in accelerating the reaction Pyruvate + NADH ----$->$ Lactate $+\mathrm{NAD}^{+}+\mathrm{H}^{+}$. From Fig.4 we can clearly observe that the frequency $\mathrm{f}=0.3066(\lambda=656 \mathrm{~nm})$ determined by the RRM as the possible frequency corresponding to the activation of the enzyme sample, does influence on its biological function. Hence, the results reveal that this specific biological process can be modulated by irradiation with defined frequencies strongly supporting the main concept of the RRM methodology. Moreover, if we observe the increase of LDH activity at $650-675 \mathrm{~nm}$, it is expected that much stronger effect in protein activation will be gained if we could perform the experiment with the predicted by the RRM characteristic frequency $\mathrm{f}=0.1680 \quad(\lambda=1156)$ that correspond to the common dehydrogenase activity. Such study would be the next step of our research of protein interactions with EMF.

\section{CONCLUSION}

This study is an attempt to shed a new light on possible deeper physical grounds that lead to understanding of protein interactions. Preliminary results obtained reveal that the frequencies obtained for dehydrogenase enzymes ( $\mathrm{f}=0.3060$ and $\mathrm{f}=0.1680$ ) using the RRM approach can be directly related to the resonances in electron differential scattering cross section of these macromolecules. The presented methodology may allow the generalization of the main advantages of the RRM in the case when the space structure of macromolecules is taken into account in a more realistic way. Based on the RRM spectral characteristic we can calculate the wavelength of electromagnetic energy that can be used to modulate the protein activity hence giving rise to an innovative efficient methodology to program, predict, design and modify proteins and their bioactivity. This could have major implications in drug design, medicine, agriculture, pharmacology and biotechnology.

\section{REFERENCES}

[1] E. J. Lerner .--ed., (1984) "Biological effects of electromagnetic fields", IEEE Spectrum.

[2] T. Karu, (1987) "Photobiological fundamentals of low-power laser therapy", IEEE Journal of Quantum Electronics, QE-23: 1703-1717.

[3] T. Karu, (1999) "Primary and Secondary Mechanisms of Actions of Visible to Near-IR Radiation on Cells", J. Photochem. Photobiol, 49:1-17.

[4] H. Frohlich, (1986) "Coherent excitation in active biological systems", In: Modern Bioelectrochemistry, F. Gutmann, \& H. Keyzer, eds., New York: Plenum, 241-261.

[5] M. Ahma, A.R. Cashmore, (1993) "HY4 Gene of A. Thaliana Encodes a Protein with Characteristics of Blue-light Photoreceptor", Nature, 366:162-166.

[6] H. Blum, (1959) Carcinogenesis by Ultraviolet Light, (N.J.), Princeton University Press, Princeton.

[7] I. Cosic, (1997) "The Resonant Recognition Model of Macromolecular Bioactivity: Theory and Applications, Basel, Birkhauser Verlag.

[8] I. Cosic, (1994) "Macromolecular Bioactivity: Is it Resonant Interaction between Macromolecules?-Theory and Applications", IEEE Trans. on Biomedical Engineering, 41:1101-1114.

[9] I. Cosic, (1995) "Virtual Spectroscopy for Fun and Profit", Biotechnology, 13: 236-238.

[10] I. Cosic, S. Birch, (1994) "Photoreceptors Having Similar Structure but Different Absorptions Can be Distinguished using the Resonant Recognition Model", Proc. IEEE EMBS, 16: 265-266.

[11] I. Cosic, V. Vojisavljevic, M. Pavlovic, (1989) "The Relationship of the Resonant Recognition Model to effects of Low-intensity Light on Cell Growth", Int. J. Radiat. Biology, 56: 179-191.

[12] G. Biscar, (1976) "Photon Enzyme Activation", Bull. Math. Biology, 38:29-38.

[13] E. Pirogova,, Q. Fang,, I. Cosic, (2003) "Evaluation of the RRM model using dehydrogenase protein as example", Proc. of 5th IFAC Symposium on Modelling and Control in Biomedical Systems, 341346. 